\title{
Role of the Aspartyl-Asparaginyl- $\beta$-Hydroxylase Gene in Neuroblastoma Cell Motility
}

\author{
Paul S. Sepe, Stephanie A. Lahousse, Brad Gemelli, Howard Chang, \\ Takashi Maeda, Jack R. Wands, and Suzanne M. de la Monte \\ Departments of Medicine and Pathology, Rhode Island Hospital, Brown Medical School, Providence, Rhode Island
}

SUMMARY: Aspartyl (asparaginyl) $\beta$-hydroxylase (AAH) is overexpressed in various malignant neoplasms, and high levels of immunoreactivity mainly occur in infiltrating or metastasized tumors. In addition, AAH is abundantly expressed in normally invasive placental trophoblastic cells. These observations led to the hypothesis that AAH may have a role in motility and aggressive behavior of tumor cells. The present study demonstrates that AAH is overexpressed in primary human malignant neuroectodermal tumors, including medulloblastomas and neuroblastomas, and that AAH expression is at a low level or undetectable in the normal mature brain. In the Sy5y neuroblastoma cell line, endogenous expression of the 86-kd AAH protein was demonstrated by Western blot analysis, and immunoreactivity predominantly localized to the cell surface by immunocytochemical staining and FACS analysis. Sy5y cells that were stably transfected with the human AAH cDNA had increased levels of proliferating cell nuclear antigen and Bcl-2, and reduced levels of p21/Waf1 and p16. In addition, increased AAH expression enhanced Sy5y cell motility, whereas antisense oligodeoxynucleotide inhibition of AAH significantly reduced Sy5y cell motility and increased the levels of p21/Waf1 and p16. The findings suggest that AAH overexpression contributes to the malignant phenotype of neuroectodermal tumor cells by increasing motility and enhancing proliferation, survival, and cell cycle progression. Because $\mathrm{AAH}$ expression is at a low level or undetectable in normal brain, the AAH gene may be a target for treating primitive neuroectodermal tumors. (Lab Invest 2002, 82:881-891).

$M$ alignant (primitive) neuroectodermal tumors are often highly fatal as a result of extensive tissue infiltration and metastatic spread. Research conducted within the last decade demonstrated that specific adhesion molecules or receptors such as CD44 (Merzak et al, 1994; Radotra and McCormick, 1997; Radotra et al, 1994) and integrins (Goldbrunner et al, 1996, 1998; Paulus et al, 1996; Tysnes et al, 1996), as well as matrix degrading/modifying enzymes such as hyaluronidase (Liu et al, 1996; Pilkington, 1996) and metalloproteinases (MMPs) (Apodaca et al, 1990; Giese and Westphal, 1996; Nakano et al, 1995; Pilkington, 1996; Sawaya et al, 1996), are critical regulators of infiltrative and metastatic tumor cell growth. In addition, a third important factor is that the recipient tissues express extracellular matrix molecules such as laminin, fibronectin, and collagen type IV that enable tumor cell attachment and infiltration (Giese et al, 1998; Goldbrunner et al, 1996; Merzak et al, 1995). Similar adhesion molecule- or receptor-mediated interactions with extracellular matrix occur during embryonic cell migration (Romanic and Madri, 1994), trophoblast implantation (Canete-Soler et al, 1995),

\section{DOI: 10.1097/01.LAB.0000020406.91689.7F}

Received April 10, 2002.

This work was supported by Grants CA35711 and AA02666 from the National Institutes of Health.

Address reprint requests to: Dr. S. M. de la Monte, Liver Research Center, Rhode Island Hospital, 55 Claverick Street, 4th Floor, Providence, RI 02903. E-mail:delamonte@hotmail.com and axonal regeneration (Fitch and Silver, 1997; Frisen, 1997; Patterson, 1985).

Infiltrative growth of malignant neoplasms may be controlled by inhibiting the expression of genes that encode matrix-degrading or matrix-modifying enzymes. For example, glioblastoma cell invasiveness has been linked to down-regulation or inactivation of the p16 tumor suppressor gene (Ueki et al, 1996) and increased MMP-2 expression (Rooprai and McCormick, 1997), whereas experimental restoration of $\mathrm{p} 16$ expression and down-regulation of MMP-2 inhibit glioma cell invasiveness (Mohanam et al, 1995). Moreover, tissue inhibitors of MMP (Mohanam et al, 1995; Nakano et al, 1995) can modulate the integrity of extracellular matrix and tumor cell invasion. In this regard, the human aspartyl (asparaginyl) $\beta$-hydroxylase (AAH) gene is of interest because of its potential role in regulating infiltrative or metastatic growth of malignant neoplasms through $\beta$-hydroxylation of extracellular matrix molecules or receptors that contain the required epidermal growth factor-like domain consensus sequence (Ince et al, 1997, 2000; Lavaissiere et al, 1996).

The human AAH cDNA encodes a 757-amino acid protein with a predicted $\mathrm{M}_{\mathrm{r}}$ of $\sim 86 \mathrm{kd}$ (Lavaissiere et al, 1996). AAH is a member of the $\alpha$-ketoglutarate-dependent dioxygenase family, which includes prolyl-3, prolyl-4, and lysyl hydroxylases (Jia et al, 1992; Wang et al, 1991). AAH catalyzes posttranslational hydroxylation of $\beta$ carbons of specific aspartate and asparagine residues in certain epidermal growth factor-like domains present in a number of proteins, including receptors and receptor ligands involved in cell growth 
and differentiation, and extracellular matrix molecules, such as tenascin, laminin, and thrombospondin (Lavaissiere et al, 1996). Importantly, the consensus sequence for $\mathrm{AAH}$ hydroxylation is also present in the epidermal growth domains of Notch and oncogenic Notch homologs; Notch has a demonstrated role in neuronal migration during development. $\mathrm{AAH}$ protein contains a relatively compact and highly charged carboxyl region that can be proteolytically cleaved from the amino terminal region to generate an approximately $52-k d$ or $56-k d$ catalytically active fragment (Jia et al, 1992, 1994; Wang et al, 1991). Site-directed mutagenesis studies demonstrated that the ${ }^{675} \mathrm{His}$ residue present in the $\mathrm{C}$-terminal fragment is essential for catalytic activity (Dinchuk et al, 2000; Jia et al, 1992) and that the ${ }^{675} \mathrm{His} \rightarrow$ Ala mutated human $\mathrm{AAH}$ cDNA exerts a dominant-negative phenotype with respect to $\mathrm{AAH}$ activity in transfected cells (Dinchuk et al, 2000; Ince et al, 2000).

We previously demonstrated AAH overexpression in hepatocellular carcinomas, cholangiocarcinomas, and adenocarcinomas of breast or lung origin and low to undetectable $\mathrm{AAH}$ expression in normal tissue counterparts (Lavaissiere et al, 1996). Further studies revealed that $\mathrm{AAH}$ overexpression was linked to malignant transformation rather than regeneration or benign proliferation of intrahepatic bile ducts (Ince et al, 2000). Clues regarding the potential role of $\mathrm{AAH}$ in cellular transformation stemmed from our investigations of a broad range of immunohistochemically stained normal and neoplastic tissues that disclosed high levels of AAH mainly in infiltrated and metastasized tumors and in normally invasive trophoblastic cells (Lavaissiere et al, 1996). These observations suggested a link between AAH overexpression and cellular invasiveness. The present study characterizes AAH expression in primary human primitive (malignant) neuroectodermal tumors of central nervous system (CNS) or peripheral origin, because these neoplasms cause death mainly by tissue infiltration and metastatic spread. In addition, we conducted experiments to examine the potential role of $\mathrm{AAH}$ in motility and aggressive growth of neuroblastoma cells.

\section{Results}

\section{AAH Immunoreactivity in Surgical Biopsy Specimens of Human Malignant Neuroectodermal Tumors}

AAH immunoreactivity was examined in human primitive neuroectodermal tumor (PNET) biopsy specimens subcategorized as supratentorial neuroblastoma ( $n=8)$, peripheral (sympathetic and non-CNS) neuroblastoma ( $n=6)$, or medulloblastoma $(n=14)$. The original hematoxylin and eosin-stained sections were reviewed to confirm the diagnoses using World Health Organization criteria (Kleihues and Cavenee, 1999). Paraffin sections containing representative samples of tumor were used in this study. Adjacent tumor-free brain tissue and normal unrelated brain biopsy specimens $(n=4)$ were included as negative controls. AAH immunoreactivity was detected using the FB-50 mAb (Lavaissiere et al, 1996), which binds to the amino terminal region of the $\mathrm{AAH}$ protein (Dinchuk et al, 2000). Immunoreactivity was revealed by the avidin-biotin horseradish peroxidase (HRP) complex method (Vector ABC Elite Kit; Vector Laboratories, Burlingame, California) using Nova Red (Vector Laboratories) as the chromogen and light hematoxylin as the counterstain. As positive and negative controls for the procedure, adjacent sections were immunostained with mAb to glial fibrillary acidic protein and hepatitis B surface antigen. All specimens were batch immunostained using the same Ab dilutions and detection reagents.

$\mathrm{AAH}$ immunoreactivity was detected in the cytoplasm and on cell membranes of all PNET specimens examined (Fig. 1, A to C) but not in normal brain (Fig. 1D). The intensity and degree of cellular labeling varied among the cases and within individual specimens, in that the most prominent immunoreactivity often occurred in tumor cells distributed at the interfaces with

(

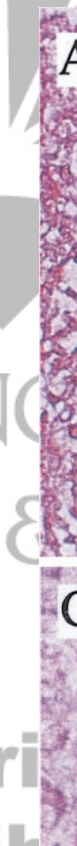

A
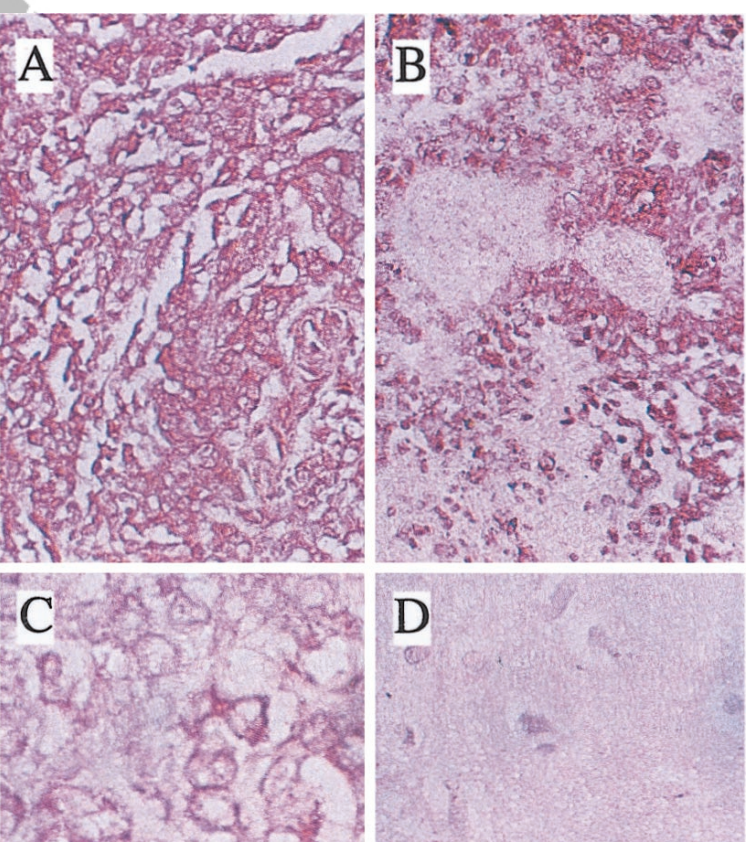

ic.

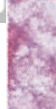

6

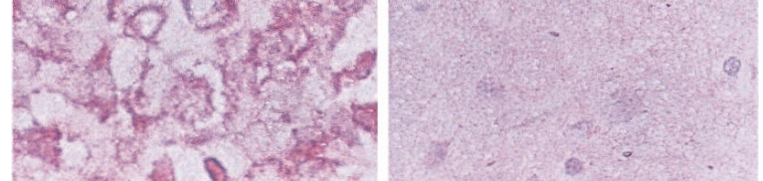

\section{Figure 1.}

Immunolocalization of aspartyl (asparaginyl) $\beta$-hydroxylase (AAH) in human malignant neuroectodermal tumors. Formalin fixed paraffin-embedded sections of primitive neuroectodermal tumors (PNETs) were immunostained to detect AAH using the FB-50 mAb. Immunoreactivity was revealed by the avidin-biotin horseradish peroxidase (HRP) method using Nova Red as the chromogen. The sections were counterstained lightly with hematoxylin. A and $B$, Low-magnification images of central nervous system-derived PNETs showing abundant $\mathrm{AAH}$ immunoreactivity in nearly all $(\mathrm{A})$ or approximately $60 \%(\mathrm{~B})$ of the neoplastic cells. The central unstained areas in panel $B$ represent foci of endothelial cell proliferation. C, High-magnification image of a PNET showing surface membrane AAH immunoreactivity. D, Intact mature cerebral cortex immunostained with the FB-50 Ab. 
relatively intact brain or in cells that had invaded the leptomeninges or subarachnoid space. The variation in distribution or intensity of $\mathrm{AAH}$ immunoreactivity could not be correlated with DNA synthesis because the percentages of nuclei in mitosis (1\% to $5 \%$ ) were similar in the central portions and infiltrating margins of the tumors. These findings correlate with observations made in non-neural malignant neoplasms in which the highest levels of $\mathrm{AAH}$ expression were detected in infiltrated or metastasized cells.

\section{AAH Expression in Sy5y Neuroblastoma Cells}

$\mathrm{AAH}$ expression was examined in Sy5y neuroblastoma cells by Western blot analysis, immunocytochemical staining, and FACS. The Sy5y cell line was cloned from a human peripheral neuroblastoma and exhibits high levels of MYCN expression in the absence of gene amplification (Sadee et al, 1987). In a subset of neuroblastomas that lack MYCN gene amplification, increased MYCN expression is mediated by HuD stabilization of MYCN mRNA transcripts (Lazarova et al, 1999). Western blot analysis with the FB-50 mAb detected the expected 86-kd AAH protein in cell lysates and higher levels of AAH in Sy5y cells compared with normal brain (Fig. 2A). In addition, the levels of $\mathrm{AAH}$ expression did not vary with duration in culture from 24 to 72 hours (Fig. 2A). Cytospin preparations of nonpermeabilized and saponinpermeabilized Sy5y cells were immunostained with the FB-50 mAb, and immunoreactivity was revealed using the avidin-biotin HRP method and diaminobenzidine as the chromogen. $\mathrm{AAH}$ immunoreactivity was localized to the surface membranes of both intact and permeabilized cells (Fig. 2B). In contrast, cells incubated with nonrelevant $A b$ to hepatitis $B$ virus exhibited negative immunostaining reactions (Fig. 2C).

FACS analysis was used to confirm the predominantly cell surface AAH expression. Dissociated nonpermeabilized and saponin-permeabilized Sy5y cells were immunolabeled with either the $\mathrm{HBOH}$ (Fig. 2D) or FB-50 (Fig. 2E) mAb to human AAH. Immunoreactivity was detected with biotinylated secondary $A b$ and fluorescein-conjugated streptavidin. Negative control labeling studies in which the primary $\mathrm{Ab}$ was omitted were conducted in parallel. FACS analysis demonstrated that the majority of cells $(>90 \%)$ exhibited surface labeling with either the $\mathrm{HBOH}$ or FB-50 Ab. With saponin permeabilization, virtually all cells exhibited $\mathrm{FB}-50$ and $\mathrm{HBOH}$ immunoreactivity, indicating that $\mathrm{AAH}$ protein was also expressed intracellularly. Of note is that the mean fluorescence intensity was higher in intact $(\mathrm{HBOH}-148.9$; FB-50-263.5) relative to permeabilized (HBOH-67.8; FB-50-179.1) cells, suggesting the presence of more abundant surface relative to intracellular AAH molecules in Sy5y cells.

\section{Effects of AAH Overexpression in Transfected Sy5y Cells}

To determine the effects of increased AAH expression on genes that are modulated with aggressive tumor behavior, Sy5y cells were transfected with a cDNA
A
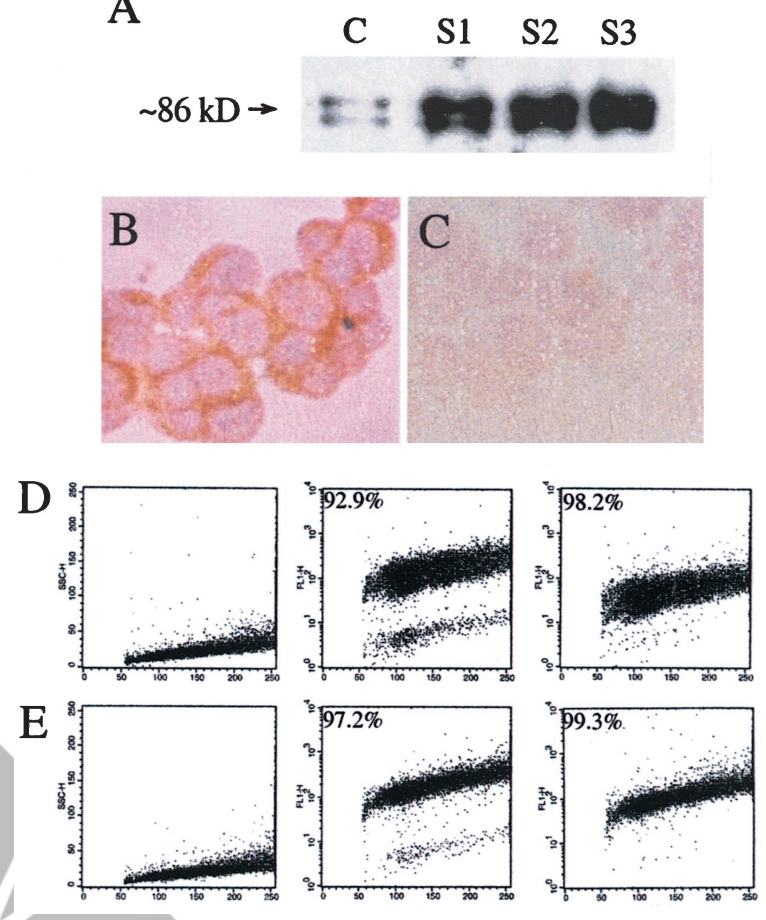

Figure 2.

AAH protein expression detected in Sy5y cells by Western blot analysis (A), immunocytochemical staining ( $B$ and $C$ ), and FACS analysis ( $D$ and $E$ ). A, For Western blot analysis, homogenates of normal brain ( $C$; cerebellar tissue) and Sy5y cells ( $S$; S1, S2, and S3 denote 1, 2, and 3 days in culture) containing 60 $\mu \mathrm{g}$ of protein were subjected to SDS-PAGE and transferred to polyvinylidene difluoride membranes. The blots were probed with the FB-50 mAb to AAH. Immunoreactivity was revealed with $\mathrm{HRP}$-conjugated secondary $\mathrm{Ab}$, ECL reagents, and film autoradiography. $B$ and $C$, Sy5y cells were fixed in Histofix, permeabilized with saponin, and immunostained with the $\mathrm{FB}-50$ (B) or a nonrelevant hepatitis $B(C)$ mAb using the $A B C$ method and diaminobenzidine

as the chromogen. Despite cell permeabilization, most of the AAH immunoreactivity was localized to surface membranes. $D$ and $E$, FACS results of $A A H$ immunoreactivity detected in Sy5y cells with $\mathrm{HBOH}(\mathrm{D})$ or $\mathrm{FB}-50$ (E) mAbs. Immunoreactivity was revealed using biotinylated secondary $A b$ and FITCconjugated streptavidin. For $D$ and $E$, the left panel depicts the profile generated with unlabeled cells, the middle panel reflects nonpermeabilized immunostained cells, and the right panel shows results obtained with saponin-permeabilized immunostained cells. The percentages of labeled cells are indicated within the panels.

containing the entire coding sequence of human $\mathrm{AAH}$ $(\mathrm{pAAH})$. The human $\mathrm{AAH}$ cDNA was ligated into a pcDNA3.1 vector in which gene expression in transfected mammalian cells was under the control of a CMV promoter. As a negative control, Sy5y cells were transfected with recombinant pcDNA3.1 carrying the luciferase reporter gene (pLuc). Five pAAH and 5 pLuc neomycin-resistant clones were studied. Increased $\mathrm{AAH}$ expression in the pAAH-transfected clones was confirmed by Western blot analysis. pLuc expression was confirmed using a standard luciferase assay.

Because aggressive behavior of malignant neoplasms is associated with increased DNA synthesis, cell cycle progression, resistance to apoptosis, motility, and invasiveness, the expression of proliferating cell nuclear antigen (PCNA), p21/Waf1, Bcl-2, and p16 was evaluated by Western blot analysis in stably transfected cells. PCNA was used as an index of DNA synthesis and cell proliferation. p21/Waf1 is a cell 
cycle inhibitor. Bcl-2 inhibits apoptosis in neuronal cells (Behl et al, 1993). p16 is an oncosuppressor gene that is often either down-regulated or mutated in infiltrating malignant neoplasms (Costello et al, 1996; He et al, 1994; Moulton et al, 1995; Ueki et al, 1996). In addition, cells were evaluated in directional motility assays using a modified Boyden chamber-type system and ATPLite luminescence to quantify cell density (see "Materials and Methods").

Cells stably transfected with $\mathrm{pAAH}$ exhibited significantly increased levels of PCNA ( 35 kd; $p<0.001)$ and $\mathrm{Bcl}-2(\sim 25 \mathrm{kd} ; p<0.05)$ and reduced levels of p21/Waf1 ( 21 kd; $p<0.001)$ and p16 ( 16 kd; $p<$ $0.001)$, although $\mathrm{AAH}$ expression was increased only by $75 \%$ to $100 \%$ relative to control (Fig. 3). Using an ATPLite luminescence-based directional motility assay and either $1 \%$ or $2 \%$ FCS as the trophic factor, we detected 2-fold or greater increases in the mean motility index of $\mathrm{pAAH}$ relative to pLuc-transfected control cells ( $p<0.001$; Fig. 4). Therefore, even modest degrees of $\mathrm{AAH}$ overexpression can enhance cellular functions associated with aggressive malignant behavior of neuroblastomas.

\section{AAH Antisense Oligodeoxynucleotide (ODN) Studies}

To further investigate the role of $\mathrm{AAH}$ as a mediator of cell motility, we inhibited $\mathrm{AAH}$ expression using antisense ODNs. Three 20-mer antisense ODNs, one sense ODN, and one nonrelevant ODN were used in our studies (Fig. 5A). The antisense and sense ODNs targeted the initiation codon of human AAH mRNA. To demonstrate specific inhibition of $\mathrm{AAH}$ expression, in vitro transcription/translation assays were performed using the TNT Quick Coupled Transcription/Translation System (Promega, Madison, Wisconsin). The translated products were labeled with $\left[{ }^{35}\right.$ S]methionine and analyzed by SDS-PAGE and autoradiography. In vitro transcription/translation studies demonstrated expression of the expected $\sim 86-\mathrm{kd}$ AAH protein using the human AAH cDNA as a template (Fig. 5B). Inclusion of antisense AAH ODN in the reactions produced dose-dependent reductions in $\mathrm{AAH}$ expression, whereas the same concentrations of sense or nonrelevant ODN had no effect on the levels of $A A H$ protein synthesized (Fig. 5B). Inhibition of AAH was achieved with each of the antisense ODNs used.

To examine the effects of antisense ODN inhibition of $\mathrm{AAH}$ expression in vivo, Sy5y cells were transfected with Location -6 AAH antisense, sense, or nonrelevant ODN using streptolysin O (SLO) permeabilization (Barry et al, 1993; Spiller and Tidd, 1995; Spiller et al, 1998b). AAH expression was examined using the microtiter immunocytochemical ELISA (MICE) assay (de la Monte et al, 1999) and Western blot analysis, and the effects of $\mathrm{AAH}$ antisense on cell migration were evaluated with the ATPLite-based directional motility assay as described (see "Materials and Methods"). To demonstrate ODN uptake, SLOpermeabilized cells were incubated with FITC endlabeled ODNs. Cells were subsequently labeled with propidium iodide and fixed with $2 \%$ paraformalde-
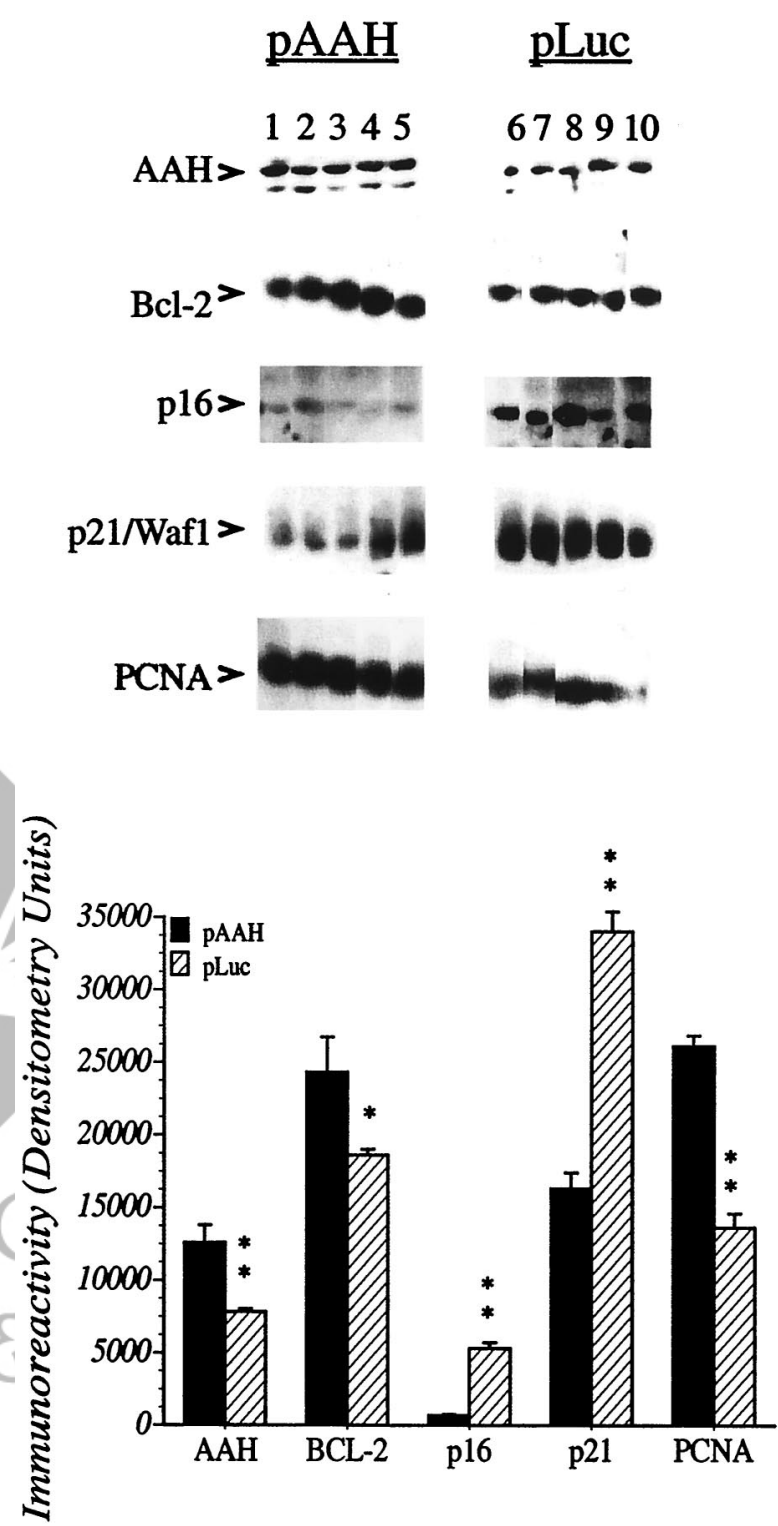

Figure 3.

Effects of AAH overexpression on the levels of Bcl-2, p16, p21/Waf1, and proliferating cell nuclear antigen (PCNA) in Sy5y cells. Sy5y cells were stably transfected with the full-length human cDNA encoding human AAH ( $p A A H)$ or luciferase $(p L u c)$. AAH gene expression was under the control of a CMV promoter. Stable pAAH (lanes 1 to 5) and pLuc-negative control (lanes 6 to 10) neomycin-resistant clones were studied. Western blot analysis was performed with cell lysates containing $60 \mu \mathrm{g}$ of protein. Replicate blots were probed with the different antibodies. Representative autoradiographs are shown above. Immunoreactivity was measured by densitometric analysis of nonsaturated autoradiographs. The bar graphs depict mean \pm SD of protein levels measured in three experiments. All differences are statistically significant by $t$ test analysis $\left({ }^{\star} p<0.05 ;{ }^{* *} p<0.001\right)$

hyde. FACS analysis demonstrated FITC and propidium iodide colocalized in $100 \%$ of cells (Fig. 6A). Additional time-course analyses revealed intracellular persistence of the FITC-labeled phosphodiesterlinked ODN for up to 8 hours (data not shown). In comparison, only $10 \%$ to $20 \%$ of cells were successfully transfected with ODNs using calcium phosphate, cationic lipids, or polyamine reagents (not shown). Although phosphorothioate-linked ODNs have longer 


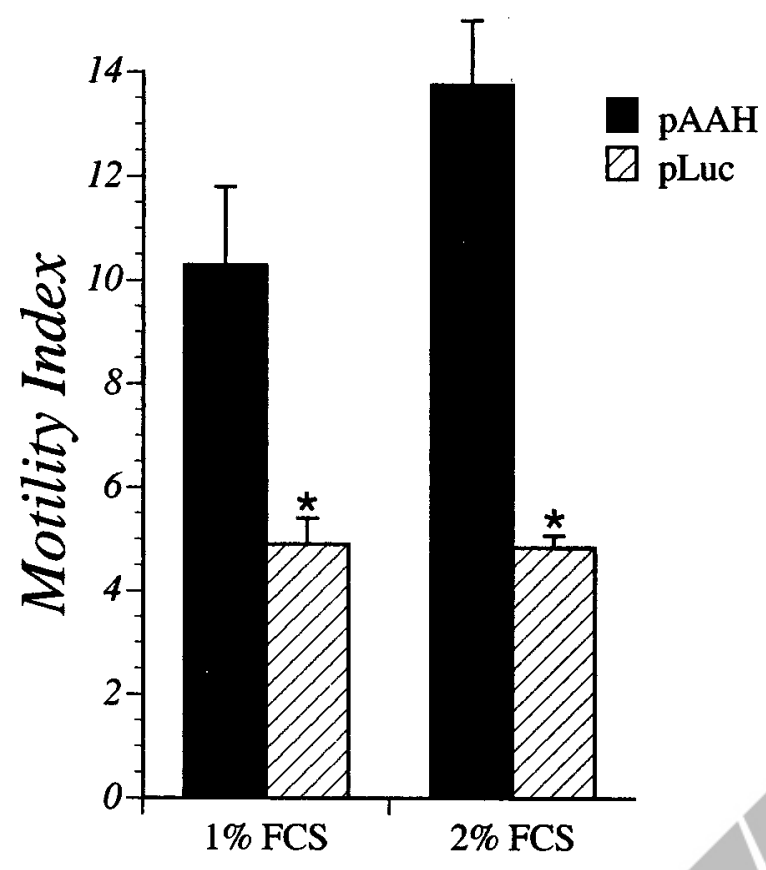

Figure 4.

Sy5y cell motility correlates with AAH expression. Sy5y cells were stably transfected with the full-length human cDNA encoding $\mathrm{AAH}(p A A H)$ or luciferase $(p L u c)$. Stable pAAH $(n=5)$ and pLuc $(n=5)$ negative control neomycin-resistant clones were analyzed. Directional motility was measured using Boyden chamber-type cell culture inserts and an ATPLite luminescencebased assay to determine the percentages of motile cells (Motility Index) in each assay chamber (see "Materials and Methods"). The ATPLite luminescence-based assay enables enumeration of all cells (nonmotile, motileadherent, and motile-nonadherent) within an assay chamber, and in preliminary studies we demonstrated a linear relationship between cell number and ATPLite luminescence in the range of cell densities used in the assays (not shown). FCE ( $1 \%$ or $2 \%$ ) was used as the trophic stimulus for cell migration. The graph depicts the mean $( \pm \mathrm{SD})$ percentages of cells that migrated through $8-\mu \mathrm{M}$ porous membranes to the bottoms of the wells (migrated-nonadherent) within a 2-hour incubation period $\left({ }^{*} p<0.001\right)$.

intracellular half-lives because of nuclease resistance, in preliminary studies we detected better inhibition and specific binding with phosphodiester-linked $\mathrm{AAH}$ antisense ODNs. Therefore, the in vivo studies were conducted using phosphodiester-linked ODNs.

Transfection of Sy5y cells with $\mathrm{AAH}$ antisense ODN resulted in substantial reductions in $\mathrm{AAH}$ protein expression relative to cells transfected with $\mathrm{AAH}$ sense or nonrelevant ODN, as demonstrated by Western blot analysis and the MICE assay (Fig. 6, B and C). In contrast, expression of glyceraldehyde-3-phosphate dehydrogenase (nonrelevant) was unaffected by ODN transfection (not shown). Sy5y cells transfected with $\mathrm{AAH}$ antisense ODNs exhibited reduced directional motility, manifested by significant reductions in the mean percentages of migrated-adherent (46\%; Fig. 6D), migrated-nonadherent (55\%; Fig. 6E), and total migrated (52\%; Fig. 6F) cells relative to control cells transfected with AAH sense or nonrelevant ODN (all $p$ $<0.001)$. MICE assays were used to measure PCNA, $\mathrm{Bcl}-2, \mathrm{p} 21$, and p16 expression in cells transfected with ODNs. Those studies demonstrated significantly increased levels of p21 and p16 expression but no
A

\begin{tabular}{ll|}
\hline \multicolumn{2}{|c|}{ Oligodeoxynucleotide Sequences Used in Experiments } \\
AAH Antisense Location (-1) & 5' CATTCTTACGCTGGGCATT \\
AAH Antisense Location (-6) & 5' TTACGCTGGGCCATTGCACG \\
AAH Antisense Location (-11) & 5' CTGGGCCATTGCACGGTCCG \\
AAH Sense (-8) & 5' ATCATGCAATGGCCAGCGTAA \\
Non-relevant & 5' GTTTTTCTTTGAGGTTAGG \\
& \\
\hline
\end{tabular}

B

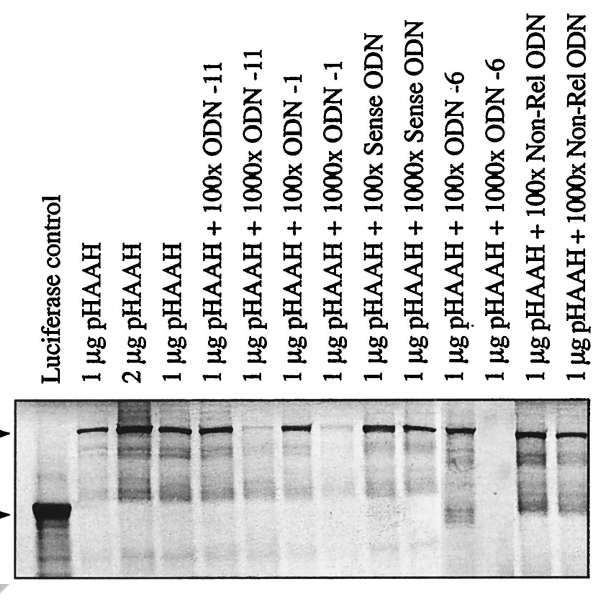

Figure 5.

In vitro transcription/translation analysis of AAH expression in the presence of AAH antisense, AAH sense, or nonrelevant (Non-Rel) oligodeoxynucleotides $(O D N s)$. A, The sequences of the antisense (Locations $-1,-6,-11$ ), sense (Location -8), and nonrelevant ODNs used in the experiments are listed. B, In vitro transcription/translation assays were performed using the TNT Quick Master Mix. $\left[{ }^{35}\right.$ S $]$ methionine-labeled products were analyzed by SDS-PAGE and autoradiography. Parallel studies were conducted in which $100 \times, 1000 \times$, or 10,000 $\times$ (not shown) molar excesses of antisense AAH, sense AAH, or nonrelevant ODN were included in the reaction. A luciferase $(L u C)$ CDNA construct was used as a positive reaction control.

alterations in either PCNA or Bcl-2 expression in cells transfected with AAH antisense ODN (Fig. 7).

\section{Discussion}

The studies described herein demonstrate that $A A H$ is abundantly expressed in primary human malignant neuroectodermal tumors and that in Sy5y cells, constitutively increased $\mathrm{AAH}$ expression is associated with increased levels of PCNA and $\mathrm{Bcl}-2$, reduced levels of p21/Waf1 and p16, and enhanced motility in a directional migration assay. Moreover, experiments showed that antisense ODN inhibition of AAH substantially reduced motility and increased expression of p21 and p16 in Sy5y cells. These observations suggest that overexpression of $\mathrm{AAH}$ may contribute to the transformed phenotype of neuroblastic tumor cells by: (a) promoting proliferation and cell cycle progression; (b) inhibiting apoptosis; and (c) enhancing tumor cell migration. The experimental results support the hypothesis that $\mathrm{AAH}$ functions to promote infiltrative or metastatic growth of tumor cells.

All 28 of the primary human malignant neuroectodermal tumor specimens examined exhibited increased $\mathrm{AAH}$ immunoreactivity relative to normal 

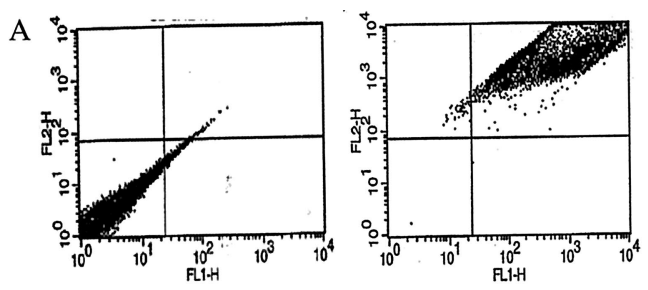

B

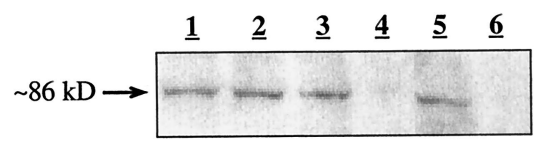

D

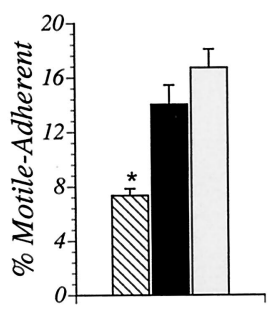

E

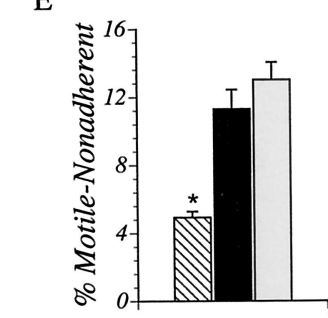

C

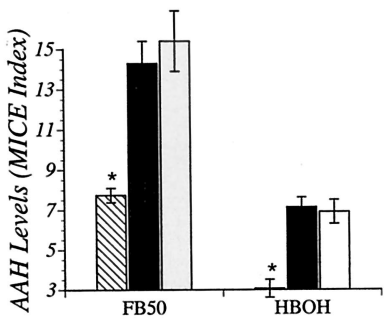

F

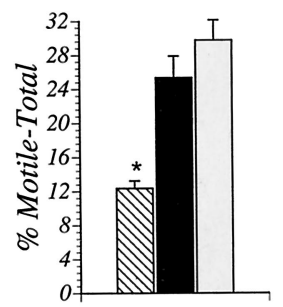

Figure 6.

Antisense ODN inhibition of AAH expression and cell motility. Sy5y cells were permeabilized using streptolysin 0 (SLO) to effect ODN uptake. A, FACS analysis demonstrating ODN uptake in 100\% of SLO-permeabilized Sy5y cells. The profile of unlabeled cells is shown at the left, and the FACS profile of cells incubated with FITC-labeled ODN and then labeled with propidium iodide is shown at the right. All cells were double-labeled with FITC and propidium iodide. B, Western blot analysis of Sy5y cells permeabilized with SLO and incubated with no DNA (lanes 1 and 5), sense ODN (lane 2), nonrelevant hepatitis B core gene ODN (lane 3), or Location -6 AAH ODN (lanes 4 and 6). C, Antisense ODN inhibition of AAH protein expression demonstrated using the microtiter immunocytochemical ELISA (MICE) assay. Immunoreactivity was detected with the $\mathrm{FB}-50$ or $\mathrm{HBOH}$ mAbs, which recognize distinct epitopes of human AAH protein. Immunoreactivity detected by the MICE assay was corrected for cell density and more sensitive than Western blot analysis ( ${ }^{*} p<0.005$ relative to other groups by ANOVA). D to F, Antisense AAH inhibition of directional motility. Directional motility was measured using Boyden chamber-type cell culture inserts and an ATPLite luminescence-based assay to quantify cell density in the upper chamber (nonmigrated), attached to the membrane outside the chamber (migrated-adherent), and at the bottom of the well (migratednonadherent). The percentages of migrated adherent (D), migrated-nonadherent (E), and total migrated (adherent + nonadherent) $(\mathrm{F})$ cells were calculated for six replicate assays per group, and the results are depicted in the graphs $\left({ }^{\star} p<0.001\right.$ relative to other control groups by ANOVA).

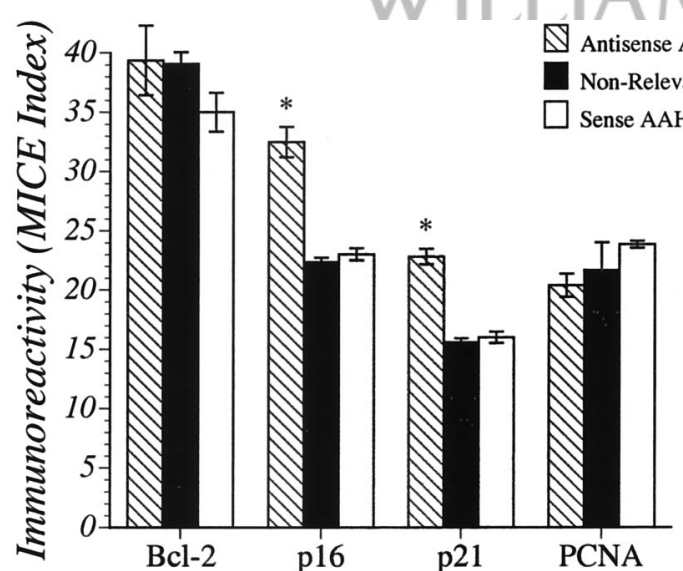

Figure 7.

Effects of antisense AAH on the levels of Bcl-2, p16, p21/Waf1, and PCNA in Sy5y cells. Sy5y cells were transfected with antisense, nonrelevant, or sense ODNs using the SLO permeabilization protocol (see "Materials and Methods"). Immunoreactivity was measured in 24 replicate culture wells using the MICE assay in which results were corrected for differences in cell density $\left({ }^{*} p<\right.$ 0.005 relative to control groups by ANOVA).

brain, suggesting that $\mathrm{AAH}$ is frequently overexpressed in these tumors. In previous studies we demonstrated that $\mathrm{AAH}$ was abundantly expressed in all infiltrative intrahepatic cholangiocarcinomas and in the majority of metastasized lung, breast, or colon carcinomas examined (Lavaissiere et al, 1996); further investigations linked $\mathrm{AAH}$ overexpression to cellular transformation (Ince et al, 2000). The findings of abundant AAH immunoreactivity in infiltrated or metastasized tumors and in normally invasive placental trophoblastic cells led to the hypothesis that $\mathrm{AAH}$ may function in cell motility as required for tumor cell invasion and metastasis. In support of this hypothesis, we demonstrated that (a) neuroblastoma cells that overexpress $\mathrm{AAH}$ exhibit significantly increased motility and (b) antisense inhibition of AAH reduces neuroblastoma cell motility.

The results further indicate that $\mathrm{AAH}$ overexpression may contribute to the malignant behavior of neuroblastoma cells by promoting growth or proliferation, survival, and cell cycle progression, as evidenced by the attendant significantly increased expression of PCNA and Bcl-2 and reduced levels of p21/Waf1 and p16. Reduced p16 expression in cells that overexpressed $\mathrm{AAH}$ is of particular interest because previous studies demonstrated that p16 modulates MMP expression (Chintala et al, 1997), and deletion mutation of the p16 gene correlates with invasive growth and rapid progression of malignant neoplasms including those of CNS origin (Moulton et al, 1995). Recent 
evidence indicates that in CNS neoplasms, p16 downregulation can occur as a result of hypermethylation of the promoter region rather than gene deletion (De Souza Nascimento et al, 2001; Nakamura et al, 2001). Reduced p16 levels may represent an additional mechanism by which malignant neoplastic cells are rendered more motile or invasive in association with AAH overexpression.

Recent metabolic labeling studies performed in our laboratory demonstrated that the half-life of $\mathrm{AAH}$ protein in Sy5y cells is $\sim 30$ minutes, suggesting that the effects of $\mathrm{AAH}$ can be rapidly modulated as might be expected for microsomal enzymes. Because the half-lives of Bcl-2 and PCNA exceed 12 hours (Kitada et al, 1993; Sasaki et al, 1995; Timchenko et al, 1996), sustained alteration in AAH gene expression may be necessary to modulate the levels of these proteins. In this regard, it is noteworthy that our experiments were conducted using phosphodiester-linked ODNs that could be detected within transfected cells for only 6 to 8 hours. In this regard, p21/Waf1 and p16 have relatively short half-lives ( $<30$ minutes) (Schwaller et al, 1997; Timchenko et al, 1996; Vijayakumar and Morrison, 1986), and correspondingly we were able to detect significant increases in the levels of these proteins after antisense AAH ODN transfection. These results are consistent with our hypothesis that $\mathrm{AAH}$ overexpression contributes to the malignant phenotype by modulating the expression of genes that regulate cell cycle progression or invasive growth.

The role of AAH as a mediator or facilitator of tumor cell migration was directly assessed in Sy5y cells transfected with the human AAH cDNA. We demonstrated a 2-fold or greater increase in the mean motility indices of cells that overexpressed $\mathrm{AAH}$ üsing Boyden chamber-type cell culture inserts and an ATPLitebased luminescence assay to quantify cell density. Complementary experiments demonstrated that transfection with antisense ODNs significantly inhibited both AAH expression and motility in Sy5y cells. Importantly, antisense inhibition of AAH significantly but proportionally decreased the percentages of migrated-adherent and migrated-nonadherent cells in each assay chamber. This suggests that $A A H$ does not selectively influence cell adhesion and instead primarily modulates motility in Sy5y cells. These results support the hypothesis that AAH has a functional role in cell migration.

Most mammalian cell types take up ODNs by highly inefficient endocytic mechanisms. Moreover, successful intracellular uptake is accompanied by rapid sequestration of ODNs into vesicles, thereby separating them from target mRNAs. In contrast, ODNs introduced directly into the cytoplasm by microinjection, rapidly accumulate in the nucleus. One method developed to improve ODN delivery into the cytoplasm involves SLO treatment. SLO reversibly permeabilizes the plasma membrane and thereby enables a biochemical-type "microinjection" of ODNs directly into the cytoplasm (Barry et al, 1993; Spiller and Tidd, 1995). SLO permeabilization of myelogenous leukemia cells resulted in the accumulation of more than 100- fold higher intracellular levels of ODNs, with substantial internalization into nuclei and specific suppression of target mRNA compared with other methods of ODN delivery (Spiller et al, 1998a, 1998b). Using the SLO method, we achieved 100\% ODN delivery into Sy5y neuroblastoma cells as demonstrated by FACS analysis. Because of the highly efficient method of ODN delivery, we showed that antisense inhibition of $A A H$ substantially reduced neuroblastoma cell motility.

Our experimental results suggest that AAH has an important role in neuroblastoma cell motility, which is required for infiltrative and metastatic growth of these tumors. The low levels of AAH present in normal brains makes $\mathrm{AAH}$ attractive for targeted gene disruption in a therapeutic setting. Future investigations will extend this research to experimental animal models to validate the role of AAH in neuroblastoma cell invasion in vivo. Successful application of antisense ODNs to specifically inhibit gene expression in mammalian cells has been reported by a number of investigators using both in vitro and in vivo models (Broughton et al, 1997; Clark et al, 1999; Dinchuk et al, 2000; Faria et al, 2001; Wagner, 1994; Wakita and Wands, 1994; Zimrin et al, 1996). Previous limitations surrounding the in vivo use of antisense gene therapy were a result of low physiologic stability, slow cellular uptake, and inadequate tissue specificity of the ODNs. These problems have been largely circumvented by the use of compounds that have greater nuclease resistance combined with enhanced target specificity, such as phosphoramidate-linked (Faria et al, 2001) or 2'-O-(2-methoxy)ethyl-linked (Zhang et al, 2000) antisense ODNs. Continued rapid development of the chemistry required to enhance the stability and binding specificity of ODNs will augment our capacity to selectively disrupt gene expression associated with the malignant phenotype. Alternatively, the finding of abundant AAH immunoreactivity on the cell surface as demonstrated by FACS analysis suggests that immunotargeting of surface AAH epitopes by "humanized" mAb or high-affinity single-chain Ab fragments (Boder et al, 2000) offers another potential therapeutic approach to malignant neuroectodermal tumors.

\section{Materials and Methods}

\section{Cell Lines}

Sy5y neuroblastoma cells were maintained in DMEM supplemented with $9 \mathrm{gm} / \mathrm{L}$ glucose, $4 \mathrm{~mm}$ glutamine, $10 \mu \mathrm{m}$ nonessential amino acid mixture, and $10 \%$ heat inactivated FCS. Preliminary studies demonstrated expression of AAH mRNA transcripts $(\sim 4.3 \mathrm{~Kb}$ and 2.6 $\mathrm{Kb})$ and protein $(\sim 86 \mathrm{kd})$ in Sy5y cells but not in normal adult brain. Sy5y cells were used to evaluate the effects of AAH overexpression on genes that promote cell cycle progression, survival, and proliferation. In addition, the effects of AAH gene transfection and antisense ODN inhibition of $A A H$ were examined in relation to cell motility. 


\section{Western Blot Analysis}

Cell lysates were prepared in radioimmunoprecipitation assay buffer containing protease inhibitors (Ausubel et al, 2000). The supernatants obtained after centrifuging the samples at $12,000 \times g$ for 15 minutes at $4^{\circ} \mathrm{C}$ were used for Western blot analysis. Protein concentration was measured using the bicinchoninic acid assay. Samples containing $60 \mu \mathrm{g}$ of protein were fractionated by SDS-PAGE, transferred to polyvinylidene difluoride Immobilon-P membranes (Millipore Corporation, Bedford, Massachusetts), and subjected to Western blot analysis. Immunoreactivity was detected with HRP-conjugated IgG (Pierce, Rockford, Illinois), with SuperSignal enhanced chemiluminescence reagents, and with either film autoradiography or the Kodak Digital Science Imaging Station (NEN Life Sciences Products, Boston, Massachusetts).

\section{MICE Assay}

The MICE assay (de la Monte et al, 1999) was used to measure in situ protein expression. Cells seeded into 96-well plates were fixed overnight in Histofix (Amresco, Solon, Ohio), permeabilized with $0.05 \%$ saponin, and then treated with $0.06 \%$ hydrogen peroxide to quench endogenous peroxidase activity. Nonspecific binding sites were masked with Superblock/TBS. The cells were incubated overnight at $4^{\circ} \mathrm{C}$ with primary Ab. Immunoreactivity was detected with HRPconjugated IgG and the TMB colorimetric substrate (Pierce) according to the manufacturer's protocols. TMB absorbances were measured in a Spectracount microplate reader. The levels of immunoreactivity were corrected for cell density by subsequently staining the cells with Coomassie blue dye and measuring the absorbances $(540 \mathrm{~nm}$ ) of dye eluted into $1 \%$ SDS. Previous studies demonstrated that Coomassie blue absorbance was linearly correlated with cell density between $10^{4}$ and $5 \times 10^{5}$ cells per well. The MICE index was calculated from the ratio of immunoreactivity to Coomassie blue absorbance for each well. Results from 8 to 16 replicate culture wells were statistically analyzed.

\section{Immunostaining}

Cells were fixed with Histofix (Amresco), permeabilized with saponin $(0.05 \%)$, and treated with $0.06 \%$ $\mathrm{H}_{2} \mathrm{O}_{2}$ in methanol to quench endogenous peroxidase activity. Nonspecific binding was blocked with 2\% normal serum. The cells were then incubated with primary $\mathrm{Ab}$ overnight at $4^{\circ} \mathrm{C}$ in humidified chambers. Immunoreactivity was detected with biotinylated secondary $\mathrm{Ab}$ and either fluorochrome- or HRPconjugated streptavidin. HRP activity was detected with NovaRed or diaminobenzidine (Vector Laboratories). As negative controls, identical specimens were incubated with nonrelevant primary $\mathrm{Ab}$ or with the primary $\mathrm{Ab}$ omitted.

\section{FACS Analysis}

Dissociated Sy5y cells were fixed in buffered 2\% formaldehyde. AAH immunoreactivity was examined in both intact and permeabilized cells. Cell permeabilization was achieved by treatment with saponin $(0.05 \%)$ in PBS for 10 minutes. The cells were incubated with FB-50 or $\mathrm{HBOH}$ (a kind gift from Dr. J. E. Dinchuk) mAb to AAH (Dinchuk et al, 2000), followed by biotinylated secondary $A b$ and FITC-conjugated streptavidin. The cells were subjected to FACS analysis using a Becton Dickinson FACScan (BD Immunocytometry Systems, San Jose, California).

\section{Directional Motility Assay}

Directional motility was measured using Boyden chamber-type culture inserts that had $8-\mu \mathrm{m}$ pore membranes. The chambers were seated in 24-well plates. Viable cells $\left(10^{5}\right)$ were placed into the upper chamber in serum-free medium. Medium supplemented with $1 \%$ or $2 \%$ FCS was placed in the well below to provide a trophic stimulus for migration. Migration was allowed to proceed for 4 hours. ATPLite (Packard Instrument Company, Meriden, Connecticut) was used to quantify viable cell density on the upper surface of the membrane (nonmotile), the undersurface of the membrane (migrated-adherent), and at the bottom of the well (migrated-nonadherent). Briefly, nonmotile cells were removed from the upper surface of the membrane using a cotton swab. The cells were lysed by immediately submerging the swabs into 200 $\mu \mathrm{l}$ of diluted ATP lysis solution in a well of a black 96-well microplate. Completeness of cell harvesting was monitored microscopically. Cells adherent to the undersurface of the membrane were harvested and lysed by submerging the wiped membrane in $200 \mu$ l of diluted ATP lysis solution in a second well of a black microplate. Cells in the well below the culture insert were resuspended and added directly to $25 \mu \mathrm{l}$ of undiluted ATP lysis solution in a third well of a black microplate. After a 5-minute incubation with agitation to ensure complete celf lysis, ATPLite substrate $(25 \mu \mathrm{l})$ was added to each well. The reactions were mixed for 2 minutes by gentle platform agitation. Subsequently, the plates were dark adapted for 5 minutes, and then luminescence was measured in a TopCount Microplate reader (Packard Instrument Company). The percentages of motile-adherent, motile-nonadherent, and total population of motile cells were calculated per chamber. For each experiment, replicates of four to six assays were performed, and the results were statistically analyzed. In preliminary studies, we demonstrated that ATPLite luminescence increases linearly with cell number between $10^{3}$ and $5 \times 10^{5}$ cells, as indicated by the manufacturer.

\section{Transfection of Sy5y Cells with the AAH cDNA}

The full-length human AAH cDNA (pAAH) (Lavaissiere et al, 1996) was ligated into the pcDNA3.1 expression vector in which gene expression was under the control of a CMV promoter (Invitrogen Corporation, San Di- 
ego, California). As negative controls, Sy5y cells were transfected with recombinant pcDNA3.1 containing pLuc. The cells were transfected using Cellfectin reagent (Gibco BRL, Grand Island, New York). Neomycin-resistant clones in which AAH expression was constitutively increased relative to cells transfected with pLuc were studied. Increased expression of $\mathrm{AAH}$ was confirmed by Western blot analysis and the MICE assay.

\section{AAH Reagents}

The $\sim 2.3 \mathrm{~Kb}$ coding region of the human $\mathrm{AAH}$ cDNA was ligated into the pcDNA3.1 vector (Invitrogen, Carlsbad, California) in which gene expression is regulated by a CMV promoter. AAH immunoreactivity was detected with the FB-50 mAb (Dinchuk et al, 2000; Lavaissiere et al, 1996). FB-50 binds to a core epitope comprising residues 286-291 (NPVEDS), which is amino-terminal with respect to the proteolytically processed 52-kd and $\sim 56-k d$ forms of AAH (Dinchuk et al, 2000). The HBOH1 and HBOH2 mAbs bind, respectively, to epitopes comprising residues $573-579$ (QPWWTPK) and residues 613-620 (LPEDENLR) (Dinchuk et al, 2000). Both $\mathrm{HBOH}$ antibodies were used for the FACS studies.

\section{Statistical Analysis}

Data depicted in the graphs represent means \pm SD generated with results from 3 to 16 replicate assays. All experiments were repeated at least three times. Between-group comparisons were made using Student $t$ tests or ANOVA with Fisher's least significant difference post-hoc test for significance. $p$ values less than 0.05 were considered significant.

\section{References}

Apodaca G, Rutka JT, Bouhana K, Berens ME, Giblin JR, Rosenblum ML, McKerrow JH, and Banda MJ (1990). Expression of metalloproteinases and metalloproteinase inhibitors by fetal astrocytes and glioma cells. Cancer Res 50: 2322-2329.

Ausubel FM, Brent R, Kingston RE, Moore DD, Seidman JG, Smith JA, and Struhl K (2000). Current protocols in molecular biology. New York: John Wiley \& Sons.

Barry EL, Gesek FA, and Friedman PA (1993). Introduction of antisense oligonucleotides into cells by permeabilization with streptolysin O. Biotechniques 15:1016-1018, 1020.

Behl C, Hovey LD, Krajewski S, Schubert D, and Reed JC (1993). BCL-2 prevents killing of neuronal cells by glutamate but not by amyloid beta protein. Biochem Biophys Res Commun 197:949-956.

Boder ET, Midelfort KS, and Wittrup KD (2000). Directed evolution of antibody fragments with monovalent femtomolar antigen-binding affinity. Proc Natl Acad Sci U S A 97:1070110705.

Broughton CM, Spiller DG, Pender N, Komorovskaya M, Grzybowski J, Giles RV, Tidd DM, and Clark RE (1997). Preclinical studies of streptolysin-O in enhancing antisense oligonucleotide uptake in harvests from chronic myeloid leukaemia patients. Leukemia 11:1435-1441.
Canete-Soler R, Gui YH, Linask KK, and Muschel RJ (1995). Developmental expression of MMP-9 (gelatinase B) mRNA in mouse embryos. Dev Dyn 204:30-40.

Chintala SK, Fueyo J, Gomez-Manzano C, Venkaiah B, Bjerkvig R, Yung WK, Sawaya R, Kyritsis AP, and Rao JS (1997). Adenovirus-mediated p16/CDKN2 gene transfer suppresses glioma invasion in vitro. Oncogene 15:2049-2057.

Clark RE, Grzybowski J, Broughton CM, Pender NT, Spiller DG, Brammer CG, Giles RV, and Tidd DM (1999). Clinical use of streptolysin-O to facilitate antisense oligodeoxyribonucleotide delivery for purging autografts in chronic myeloid leukaemia. Bone Marrow Transplant 23:1303-1308.

Costello JF, Berger MS, Huang HS, and Cavenee WK (1996). Silencing of p16/CDKN2 expression in human gliomas by methylation and chromatin condensation. Cancer Res 56: 2405-2410.

de la Monte SM, Ganju N, and Wands JR (1999). Microtiter immunocytochemical ELISA assay: A novel and highly sensitive method of quantifying immunoreactivity. Biotechniques 26:1073-1076.

De Souza Nascimento P, Maciel CM, Kawamura MT, Oliveira JA, Teixeira A, Carvalho M, and Alves G (2001). Molecular analysis of CDKN2 (p16) in gliomas associated with clinical data. Oncol Rep 8:1039-1043.

Dinchuk JE, Henderson NL, Burn TC, Huber R, Ho SP, Link J, O'Neil KT, Focht RJ, Scully MS, Hollis JM, Hollis GF, and Friedman PA (2000). Aspartyl beta-hydroxylase (Asph) and an evolutionarily conserved isoform of Asph missing the catalytic domain share exons with junctin. J Biol Chem 275:39543-39554.

Faria M, Spiller DG, Dubertret C, Nelson JS, White MR, Scherman D, Helene C, and Giovannangeli C (2001). Phosphoramidate oligonucleotides as potent antisense molecules in cells and in vivo. Nat Biotechnol 19:40-44.

Fitch MT and Silver J (1997). Glial cell extracellular matrix: Boundaries for axon growth in development and regeneration. Cell Tissue Res 290:379-384.

Frisen J (1997). Determinants of axonal regeneration. Histol Histopathol 12:857-868.

Giese A, Laube B, Zapf S, Mangold U, and Westphal M (1998). Glioma cell adhesion and migration on human brain sections. Anticancer Res 18:2435-2447.

Giese A and Westphal M (1996). Glioma invasion in the central nervous system. Neurosurgery 39:235-250, discussion $250-252$.

Goldbrunner RH, Bernstein JJ, and Tonn JC (1998). ECMmediated glioma cell invasion. Microsc Res Tech 43:250257.

Goldbrunner RH, Haugland HK, Klein CE, Kerkau S, Roosen K, and Tonn JC (1996). ECM dependent and integrin mediated tumor cell migration of human glioma and melanoma cell lines under serum-free conditions. Anticancer Res 16: 3679-3687.

He J, Allen JR, Collins VP, Allalunis-Turner MJ, Godbout R, Day RS III, and James CD (1994) CDK4 amplification is an alternative mechanism to 16 gene homozygous deletion in glioma cell lines. Cancer Res 54:5804-5807. 
Ince N, de la Monte SM, Friedman P, and Wands JR (1997). Overexpression of HAAH (human aspartyl asparaginyl hydroxylase) in bile ducts is related to malignant transformation. Hepatology 26:362A.

Ince N, de la Monte SM, and Wands JR (2000). Overexpression of human aspartyl (asparaginyl) beta-hydroxylase is associated with malignant transformation. Cancer Res 60: 1261-1266.

Jia S, McGinnis K, VanDusen WJ, Burke CJ, Kuo A, Griffin PR, Sardana MK, Elliston KO, Stern AM, and Friedman PA (1994). A fully active catalytic domain of bovine aspartyl (asparaginyl) beta-hydroxylase expressed in Escherichia coli: Characterization and evidence for the identification of an active-site region in vertebrate alpha-ketoglutaratedependent dioxygenases. Proc Natl Acad Sci USA 91:72277231.

Jia S, VanDusen WJ, Diehl RE, Kohl NE, Dixon RA, Elliston KO, Stern AM, and Friedman PA (1992). cDNA cloning and expression of bovine aspartyl (asparaginyl) beta- hydroxylase. J Biol Chem 267:14322-14327.

Kitada S, Miyashita T, Tanaka S, and Reed JC (1993). Investigations of antisense oligonucleotides targeted against bcl-2 RNAs. Antisense Res Dev 3:157-169.

Kleihues P and Cavenee WK, editors (1999). World Health Organization classification of tumours: Pathology and genetics, tumours of the nervous system. Lyon, France: IARC Press.

Lavaissiere L, Jia S, Nishiyama M, de la Monte S, Stern AM, Wands JR, and Friedman PA (1996). Overexpression of human aspartyl(asparaginyl)beta-hydroxylase in hepatocellular carcinoma and cholangiocarcinoma. J Clin Invest 98: 1313-1323.

Lazarova DL, Spengler BA, Biedler JL, and Ross RA (1999). $\mathrm{HuD}$, a neuronal-specific RNA-binding protein, is a putative regulator of $\mathrm{N}$-myc pre-mRNA processing/stability in malignant human neuroblasts. Oncogene 18:2703-2710.

Liu D, Pearlman E, Diaconu E, Guo K, Mori H, Haqqi T, Markowitz S, Willson J, and Sy MS (1996). Expression of hyaluronidase by tumor cells induces angiogenesis in vivo. Proc Natl Acad Sci USA 93:7832-7837.

Merzak A, Koochekpour S, and Pilkington GJ (1995). Adhesion of human glioma cell lines to fibronectin, laminin, vitronectin and collagen I is modulated by gangliosides in vitro. Cell Adhes Commun 3:27-43.

Merzak A, Parker C, Koochekpour S, Sherbet GV, and Pilkington GJ (1994). Overexpression of the 18A2/mts 1 gene and down-regulation of the TIMP-2 gene in invasive human glioma cell lines in vitro. Neuropathol Appl Neurobiol 20:614619.

Mohanam S, Wang SW, Rayford A, Yamamoto M, Sawaya R, Nakajima M, Liotta LA, Nicolson GL, Stetler-Stevenson WG, and Rao JS (1995). Expression of tissue inhibitors of metalloproteinases: Negative regulators of human glioblastoma invasion in vivo. Clin Exp Metastasis 13:57-62.

Moulton T, Samara G, Chung WY, Yuan L, Desai R, Sisti M, Bruce J, and Tycko B (1995). MTS1/p16/CDKN2 lesions in primary glioblastoma multiforme. Am J Pathol 146:613-619.

Nakamura M, Watanabe T, Klangby U, Asker C, Wiman K, Yonekawa Y, Kleihues P, and Ohgaki H (2001). p14ARF deletion and methylation in genetic pathways to glioblastomas. Brain Pathol 11:159-168.
Nakano A, Tani E, Miyazaki K, Yamamoto Y, and Furuyama J (1995). Matrix metalloproteinases and tissue inhibitors of metalloproteinases in human gliomas. J Neurosurg 83:298307.

Patterson PH (1985). On the role of proteases, their inhibitors and the extracellular matrix in promoting neurite outgrowth. J Physiol 80:207-211.

Paulus W, Baur I, Beutler AS, and Reeves SA (1996). Diffuse brain invasion of glioma cells requires beta 1 integrins. Lab Invest 75:819-826.

Pilkington CJ (1996). The role of the extracellular matrix in neoplastic glial invasion of the nervous system. Braz J Med Biol Res 29:1159-1172.

Radotra B and McCormick D (1997). Glioma invasion in vitro is mediated by CD44-hyaluronan interactions. J Pathol 181: $434-438$.

Radotra B, McCormick D, and Crockard A (1994). CD44 plays a role in adhesive interactions between glioma cells and extracellular matrix components. Neuropathol Appl Neurobiol 20:399-405.

Romanic AM and Madri JA (1994). Extracellular matrixdegrading proteinases in the nervous system. Brain Pathol 4:145-156.

Rooprai HK and McCormick D (1997). Proteases and their inhibitors in human brain tumours: A review. Anticancer Res 17:4151-4162.

Sadee W, Yu VC, Richards ML, Preis PN, Schwab MR, Brodsky FM, and Biedler JL (1987). Expression of neurotransmitter receptors and myc protooncogenes in subclones of a human neuroblastoma cell line. Cancer Res 47:52075212.

Sasaki K, Kürose A, Uesugi N, and Kato A (1995). Effects of cycloheximide on chromatin-bound and -unbound PCNA in HeLa cells. Oncology 52:419-421.

Sawaya RE, Yamamoto M, Gokaslan ZL, Wang SW, Mohanam S, Fuller GN, McCutcheon IE, Stetler-Stevenson WG, Nicolson GL, and Rao JS (1996). Expression and localization of $72 \mathrm{kDa}$ type IV collagenase (MMP-2) in human malignant gliomas in vivo. Clin Exp Metastasis 14:35-42.

Schwaller J, Pabst T, Koeffler HP, Niklaus G, Loetscher P, Fey MF, and Tobler A (1997). Expression and regulation of G1 cell-cycle inhibitors (p16INK4A, p15INK4B, p18INK4C, p19INK4D) in human acute myeloid leukemia and normal myeloid cells. Leukemia 11:54-63.

Spiller DG, Giles RV, Broughton CM, Grzybowski J, Ruddell CJ, Tidd DM, and Clark RE (1998a). The influence of target protein half-life on the effectiveness of antisense oligonucleotide analog-mediated biologic responses. Antisense Nucleic Acid Drug Dev 8:281-293.

Spiller DG, Giles RV, Grzybowski J, Tidd DM, and Clark RE (1998b). Improving the intracellular delivery and molecular efficacy of antisense oligonucleotides in chronic myeloid leukemia cells: A comparison of streptolysin-O permeabilization, electroporation, and lipophilic conjugation. Blood 91: $4738-4746$.

Spiller DG and Tidd DM (1995). Nuclear delivery of antisense oligodeoxynucleotides through reversible permeabilization of human leukemia cells with streptolysin O. Antisense Res Dev 5:13-21. 
Timchenko NA, Wilde M, Nakanishi M, Smith JR, and Darlington GJ (1996). CCAAT/enhancer-binding protein alpha (C/EBP alpha) inhibits cell proliferation through the p21 (WAF-1/CIP-1/SDI-1) protein. Genes Dev 10:804-815.

Tysnes BB, Larsen LF, Ness GO, Mahesparan R, Edvardsen K, Garcia-Cabrera I, and Bjerkvig R (1996). Stimulation of glioma-cell migration by laminin and inhibition by anti-alpha3 and anti-beta1 integrin antibodies. Int $\mathrm{J}$ Cancer 67:777-784.

Ueki K, Ono Y, Henson JW, Efird JT, von Deimling A, and Louis DN (1996). CDKN2/p16 or RB alterations occur in the majority of glioblastomas and are inversely correlated. Cancer Res 56:150-153.

Vijayakumar MN and Morrison DA (1986). Localization of competence-induced proteins in Streptococcus pneumoniae. J Bacteriol 165:689-695.

Wagner RW (1994). Gene inhibition using antisense oligodeoxynucleotides. Nature 372:333-335.
Wakita T and Wands JR (1994). Specific inhibition of hepatitis C virus expression by antisense oligodeoxynucleotides: In vitro model for selection of target sequence. J Biol Chem 269:14205-14210.

Wang QP, VanDusen WJ, Petroski CJ, Garsky VM, Stern AM, and Friedman PA (1991). Bovine liver aspartyl betahydroxylase: Purification and characterization. J Biol Chem 266:14004-14010.

Zhang H, Cook J, Nickel J, Yu R, Stecker K, Myers K, and Dean NM (2000). Reduction of liver Fas expression by an antisense oligonucleotide protects mice from fulminant hepatitis. Nat Biotechnol 18:862-867.

Zimrin AB, Pepper MS, McMahon GA, Nguyen F, Montesano $R$, and Maciag T (1996). An antisense oligonucleotide to the notch ligand jagged enhances fibroblast growth factorinduced angiogenesis in vitro. J Biol Chem 271:3249932502.

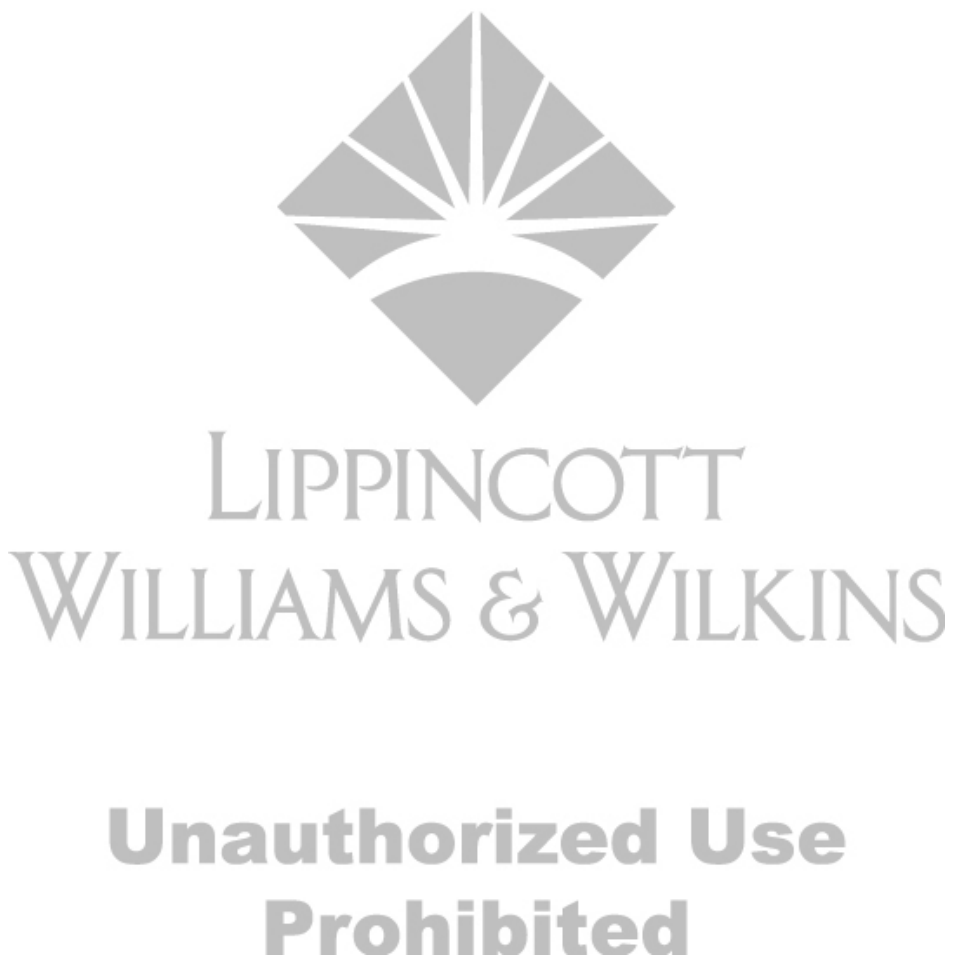

